\title{
Diagnosis of gum cancer cells from DNA/RNA using database mining and support vector regression through high resolution 4D HPCH experiment for sequential assignment of 13C-labeled DNAs/RNAs in gum cancer cells
}

\author{
Alireza Heidari ${ }^{1,2 *}$ and Ricardo Gobato ${ }^{3}$ \\ ${ }^{1}$ Faculty of Chemistry, California South University, 14731 Comet St. Irvine, CA 92604, USA \\ ${ }^{2}$ American International Standards Institute, Irvine, CA 3800, USA \\ ${ }^{3}$ Green Land Landscaping and Gardening, Seedling Growth Laboratory, 86130-000, Parana, Brazil
}

\begin{abstract}
In the current research, diagnosis of gum cancer cells from DNA/RNA using database mining and support vector regression through high resolution 4D HPCH experiment for sequential assignment of 13C-labeled DNAs/RNAs in gum cancer cells are investigated. Diagnosis of gum cancer in biopsy samples relies on morphological analysis using the expertise of histopathologists or cytologists who have many years' experience in viewing these specimens. This is augmented by techniques which allow gum cancer specific markers to be visualized using diagnosis of gum cancer cells from DNA/RNA using database mining and support vector regression through high resolution 4D HPCH experiment for sequential assignment of 13C-labeled DNAs/RNAs in gum cancer cells, where gene profiles can be visualized using microarray platforms. Characteristic translocations can be detected using fluorescent in situ hybridization (FISH), particularly in haematopathology. The use of optical methods to interrogate gum cancer cells is developing rapidly and diagnosis of gum cancer cells from DNA/RNA using database mining and support vector regression through high resolution $4 \mathrm{D} \mathrm{HPCH}$ experiment for sequential assignment of $13 \mathrm{C}-$ labeled DNAs/RNAs in gum cancer cells provides a new modality, which shows much promise.
\end{abstract}

\section{Introduction}

Diagnosis of gum cancer cells from DNA/RNA using database mining and support vector regression through high resolution $4 \mathrm{D}$ HPCH experiment for sequential assignment of 13C-labeled DNAs/ RNAs in gum cancer cells is increasingly investigated for gum cancer diagnosis. As the potential of the technique is explored and realized, it is slowly making its way into clinics. There are more reports in recent years showing promise that it can help clinicians for gum cancer diagnosis. However, a number of challenges remain to be overcome, especially in vivo gum cancer diagnosis. In this article, the recent progress of the technique toward clinical gum cancer diagnosis is discussed from a critical perspective. Diagnosis of gum cancer cells from DNA/RNA using database mining and support vector regression through high resolution $4 \mathrm{D} \mathrm{HPCH}$ experiment for sequential assignment of 13C-labeled DNAs/RNAs in gum cancer cells can provide detailed chemical information about a tissue sample and thus insight into the chemical changes that accompany gum cancer disease. In contrast to fluorescence, there are a large number of diagnosis of gum cancer cells from DNA/RNA using database mining and support vector regression through high resolution $4 \mathrm{D} \mathrm{HPCH}$ experiment for sequential assignment of 13C-labeled DNAs/RNAs in gum cancer cells active molecules in gum cancer tissue, and their spectral signatures are sharp and well delineated. The ability to measure several different chemicals is of particular importance in studying gum cancer because of the heterogeneity of the disease. Although diagnosis of gum cancer cells from DNA/RNA using database mining and support vector regression through high resolution $4 \mathrm{D} \mathrm{HPCH}$ experiment for sequential assignment of 13C-labeled DNAs/RNAs in gum cancer cells provide high information content, the signals are orders of magnitude weaker than fluorescence. However, with careful system design, collection of clinical data in relevant times with safe laser powers can be accomplished. For these reasons, we have investigated diagnosis of gum cancer cells from DNA/RNA using database mining and support vector regression through high resolution $4 \mathrm{D} \mathrm{HPCH}$ experiment for sequential assignment of 13C-labeled DNAs/RNAs in gum cancer cells as a clinical tool for the diagnosis of a variety of gum cancer pathologies [1-10].

${ }^{\star}$ Correspondence to: Alireza Heidari, Faculty of Chemistry, California South University, 14731 Comet St. Irvine, CA 92604; American International Standards Institute, Irvine, CA 3800, USA, E-mail: Scholar.Researcher.Scientist@gmail. com; Alireza.Heidari@calsu.us; Central@aisi-usa.org

Key words: diagnosis, gum cancer cells, DNA/RNA, database mining, regression, high resolution, assignment

Received: October 17, 2020; Accepted: November 16, 2020, Published: November 20, 2020 
Heidari A (2020) Diagnosis of gum cancer cells from DNA/RNA using database mining and support vector regression through high resolution 4D HPCH experiment for sequential assignment of $13 \mathrm{C}-$ labeled DNAs/RNAs in gum cancer cells

\section{Results and discussion}

Diagnosis of gum cancer cells from DNA/RNA using database mining and support vector regression through high resolution $4 \mathrm{D}$ $\mathrm{HPCH}$ experiment for sequential assignment of 13C-labeled DNAs/ RNAs in gum cancer cells has the potential to provide diagnostic information to the clinician. The technique has a number of advantages allowing individual cells to be interrogated without staining. With further developments in technology, the surgeon will be able to rapidly acquire accurate diagnostic information at the time of operation using fibre optic diagnosis of gum cancer cells from DNA/RNA using database mining and support vector regression through high resolution $4 \mathrm{D}$ $\mathrm{HPCH}$ experiment for sequential assignment of 13C-labeled DNAs/ RNAs in gum cancer cells. Improvements in signal detection and data analysis, like modulated diagnosis of gum cancer cells from DNA/RNA using database mining and support vector regression through high resolution $4 \mathrm{D} \mathrm{HPCH}$ experiment for sequential assignment of $13 \mathrm{C}-$ labeled DNAs/RNAs in gum cancer cells, will allow the rapid acquisition and analysis of spectra. There is also considerable potential in screening tissue fluids for gum cancer cells in order to facilitate early detection and for follow up after surgery for gum cancer. Collaborations between clinicians, pathologists and physicists are opening up new areas in this rapidly developing field (Figure 1).

\section{Conclusion}

Diagnosis of gum cancer cells from DNA/RNA using database mining and support vector regression through high resolution $4 \mathrm{D}$ $\mathrm{HPCH}$ experiment for sequential assignment of 13C-labeled DNAs/ RNAs in gum cancer cells has the potential to provide diagnostic

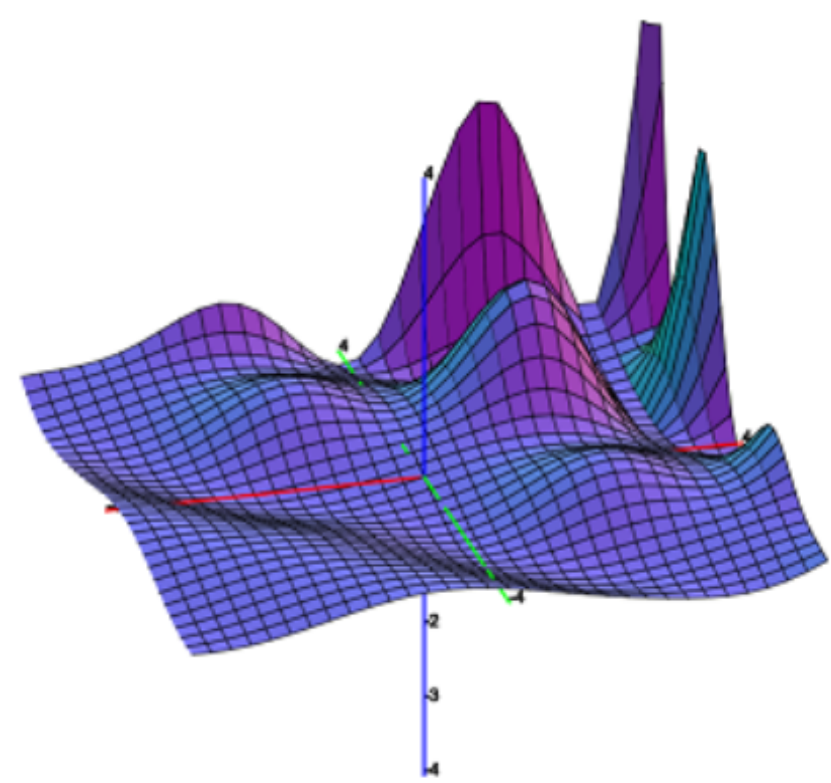

Figure 1. Diagnosis of gum cancer cells from DNA/RNA using database mining and support vector regression through high resolution $4 \mathrm{D} \mathrm{HPCH}$ experiment for sequential assignment of 13C-labeled DNAs/RNAs in gum cancer cells information to the clinician. The technique has a number of advantages allowing individual cells to be interrogated without staining. With further developments in technology, the surgeon will be able to rapidly acquire accurate diagnostic information at the time of operation using fibre optic diagnosis of gum cancer cells from DNA/RNA using database mining and support vector regression through high resolution $4 \mathrm{D}$ $\mathrm{HPCH}$ experiment for sequential assignment of 13C-labeled DNAs/ RNAs in gum cancer cells. Improvements in signal detection and data analysis, like modulated diagnosis of gum cancer cells from DNA/RNA using database mining and support vector regression through high resolution 4D HPCH experiment for sequential assignment of 13Clabeled DNAs/RNAs in gum cancer cells, will allow the rapid acquisition and analysis of spectra. There is also considerable potential in screening tissue fluids for gum cancer cells in order to facilitate early detection and for follow up after surgery for gum cancer. Collaborations between clinicians, pathologists and physicists are opening up new areas in this rapidly developing field.

\section{Acknowledgments}

This study was supported by the Cancer Research Institute (CRI) Project of Scientific Instrument and Equipment Development, the National Natural Science Foundation of the United Sates, the International Joint BioSpectroscopy Core Research Laboratory Program supported by the California South University (CSU), and the Key project supported by the American International Standards Institute (AISI), Irvine, California, USA.

\section{References}

1. Heidari A, Brown C (2015) Study of composition and morphology of cadmium oxide (CdO) nanoparticles for eliminating cancer cells. J Nanomed Res 2(5): 20.

2. Heidari A, Brown C (2015) Study of surface morphological, phytochemical and structural characteristics of rhodium (III) oxide $\left(\mathrm{Rh}_{2} \mathrm{O}_{3}\right)$ nanoparticles. International Journal of Pharmacology, Phytochemistry and Ethnomedicine 1(1): 15-19.

3. Heidari A (2016) An experimental biospectroscopic study on seminal plasma in determination of semen quality for evaluation of male infertility. Int $J$ Adv Technol 7: e007.

4. Heidari A (2016) Extraction and preconcentration of N-Tolyl-Sulfonyl-PhosphoramidSaeure-Dichlorid as an anti-cancer drug from plants: a pharmacognosy study. $J$ Pharmacogn Nat Prod 2: e103.

5. Heidari A (2016) A thermodynamic study on hydration and dehydration of DNA and RNA-Amphiphile Complexes. J Bioeng Biomed Sci S: 006.

6. Heidari A (2016) Computational studies on molecular structures and carbonyl and ketene groups' effects of singlet and triplet energies of Azidoketene $\mathrm{O}=\mathrm{C}=\mathrm{CH}-\mathrm{NNN}$ and Isocyanatoketene $\mathrm{O}=\mathrm{C}=\mathrm{CH}-\mathrm{N}=\mathrm{C}=\mathrm{O} . J$ Appl Computat Math 5: e142.

7. Heidari A (2016) Study of irradiations to enhance the induces the dissociation of hydrogen bonds between peptide chains and transition from helix structure to random coil structure using ATR-FTIR, Raman and 'HNMR Spectroscopies. J Biomol Res Ther 5: e146.

8. Heidari A (2016) Future prospects of point fluorescence spectroscopy, fluorescence imaging and fluorescence endoscopy in photodynamic therapy (PDT) for cancer cells. J Bioanal Biomed 8: e135.

9. Heidari A (2016) A bio-spectroscopic study of DNA density and color role as determining factor for absorbed irradiation in cancer cells. Adv Cancer Prev 1: e102.

10. Heidari A (2016) Manufacturing process of solar cells using cadmium oxide (CdO) and rhodium (III) Oxide $\left(\mathrm{Rh}_{2} \mathrm{O}_{3}\right)$ nanoparticles. J Biotechnol Biomater 6: e125.

Copyright: (C2020 Heidari A. This is an open-access article distributed under the terms of the Creative Commons Attribution License, which permits unrestricted use, distribution, and reproduction in any medium, provided the original author and source are credited. 